\title{
Influence of Stimulant Medication on Pedestrian Safety for Children with Excessive Daytime Sleepiness
}

\author{
Jenni B. Rouse, $\mathrm{MA}^{1}$, Kristin T. Avis, $\mathrm{PhD}^{2}$, David C. Schwebel, $\mathrm{PhD}^{1}$ \\ 'Departments of Psychology, ${ }^{2}$ Pediatrics, University of Alabama at Birmingham, Birmingham, AL, USA
}

Received: January 18, 2021

Revised: April 13, 2021

Accepted: May 12, 2021

Correspondence

David C. Schwebel, $\mathrm{PhD}$

Department of Psychology,

University of Alabama at Birmingham,

1300 University Boulevard, CH 415,

Birmingham, AL 35294, USA

Tel +1-205-934-8745

Fax +1-205-934-9896

E-mailschwebel@uab.edu

\section{ORCID}

David C. Schwebel

https://orcid.org/0000-0002-2141-8970

(C) This is an Open Access article distributed under the terms of the Creative Commons Attribution Non-Commercial License (https://creativecommons.org/licenses/by-nc/4.0) which permits unrestricted non-commercial use, distribution, and reproduction in any medium, provided the original work is properly cited.
Background and Objective Pedestrian crashes represent a leading cause of pediatric injury. Children have particular risk due to underdeveloped cognitive and executive function skills. Given children with excessive daytime sleepiness (EDS) face deficiencies in executive function and cognitive skill that impact cognitive functions necessary for safe pedestrian engagement, this study examined whether treatment with the stimulant medication modafinil might improve both cognitive functioning and pedestrian safety among children with EDS.

Methods Twelve children aged 8-16 years diagnosed with EDS participated in a within-subjects experiment. Children completed two laboratory visits, one while treated with modafinil and one untreated. During each visit, children completed two self-report sleepiness inventories, two cognitive tests [psychomotor vigilance test (PVT) and Conners' continuous performance task (CPT; version 3.0, Multi-Health Systems, Inc.)], and 20 virtual pedestrian environment street-crossings. Parents completed a sleepiness survey about children.

Results Related samples t-tests found consistent patterns of reduced sleepiness, improved cognitive functioning, and safer pedestrian behavior while children were medicated with modafinil compared to unmedicated. Statistically significant differences emerged for parent-reported child sleepiness ( $\mathrm{p}<0.01)$; PVT false starts, indicating improved attention, vigilance and alertness $(\mathrm{p}<$ $0.10)$; CPT omissions, signifying failure to respond to stimuli $(\mathrm{p}<0.05)$; and shortest time-to-contact with oncoming virtual traffic $(p<0.05)$. Chi-square demonstrated children had fewer collisions with oncoming virtual traffic while medicated than unmedicated $(\mathrm{p}<0.05)$.

Conclusions Medication with the stimulant modafinil improved multiple aspects of cognitive functioning and pedestrian safety among children with EDS. The greatest benefits emerged on measures of alertness, attention, and response latency. Results suggest modafinil may improve realworld functioning in tasks like crossing streets.

Sleep Med Res 2021;12(1):44-49

Key Words Injury and wounds; Excessive daytime sleepiness; Children, street; Modafinil; Narcolepsy.

\section{INTRODUCTION}

Pedestrian crashes resulted in 15529 nonfatal injuries and 158 deaths among American children ages 8-16 in 2018 [1]. Children are particularly susceptible to pedestrian injuries for a variety of reasons, including immature cognitive and executive function skills that lead to inattention, distraction and inability to judge, process, and act upon traffic risks $[2,3]$.

Excessive daytime sleepiness (EDS) impacts many of the same cognitive skills that are critical to safe pedestrian functioning [4]. EDS is characteristic of both idiopathic hypersomnia (IHS) and narcolepsy, neurological sleep disorders that cause individuals to be excessively sleepy despite adequate sleep at night, naps, or generally positive sleep hygiene. Individuals with EDS experience a level of sleepiness that is not remedied by napping and that impairs daily activities such as exercise, socialization, and work/academic achievement [5]. 
Relevant to pedestrian safety, children diagnosed with EDS also experience neural functioning deficits that mimic those of chronic sleep deprivation [6]. These deficits include deficiencies in executive function and cognitive skill, as well as patterns of impulsive, inattentive, and cognitively immature behavior [7,8], all skills highly relevant to safe engagement in pedestrian settings.

Individuals with EDS respond well to treatment with psychostimulant medications $[9,10]$. Thus, just as sleepy adults may selfmedicate to improve functioning with stimulants such as caffeine and individuals with diagnosed attention-deficit/hyperactivity disorder are prescribed with prescription stimulants, children with EDS experience a reduction in symptoms through stimulant medications [9]. Modafinil is one stimulant that has proven effective to reduce EDS symptoms, but it is unknown whether treatment with stimulants like modafinil also yields positive changes in real-world behavior such as risk for injury.

The present study recruited 12 children ages 8-16 diagnosed with EDS by meeting International Classification of Sleep Disorders (ICSD)-2 diagnostic criteria for a hypersomnia of central origin (narcolepsy with or without cataplexy, or IHS). A withinsubjects research design was implemented to test the children's pedestrian safety and behavior in a virtual pedestrian environment under two conditions, while untreated with medication and following their prescribed treatment with modafinil. We also evaluated cognitive skills relevant to pedestrian safety during both visits. We hypothesized the children would demonstrate safer pedestrian behaviors, plus score higher on assessment of relevant cognitive skills and lower on measures of sleepiness, while medicated with the stimulant modafinil.

\section{METHODS}

\section{Participants and Recruitment}

Twelve children [mean $(M)=13.35$ years, standard deviation $(\mathrm{SD})=2.03$ years, range $=9-16$ years] diagnosed with IHS or narcolepsy participated. The sample included 7 boys (58\%) and 5 girls (42\%), was 50\% White, 33\% African American, and 16\% multiracial/other race, and families reported a median household income of $\$ 20000-\$ 39999$ (range $<\$ 20000$ to $>\$ 100000$ ).

Participants were recruited from a Pediatric Sleep Disorders Center at major children's hospital in the United States. Eligible children met ICSD-2 diagnostic criteria for a hypersomnia of central origin (narcolepsy with or without cataplexy, or IHS) based on diagnostic assessments that included overnight polysomnography followed by multiple sleep latency tests the following day, drug screening, and thorough clinical evaluation. Exclusion criteria were minimal and included only sensory or intellectual impairments that would prevent the child from understanding or participating in the study protocol. No eligible children were excluded for these reasons.

The study protocol was approved by the Institutional Review
Board at the University of Alabama at Birmingham (IRB no. 140228005). Informed consent was obtained from participants' parents/legal guardians, and informed assent from participants. Families were compensated for their time.

\section{General Protocol}

Following informed consent processes, children completed study procedures twice, with visits separated by an average of 10.21 days ( $\mathrm{SD}=5.55$ days). One visit occurred while the child had been unmedicated by all stimulant and other wakefulness medications to treat their sleep disorder for at least 6 days. The other visit occurred while the child was treated with modafinil, as prescribed by their physician, for at least the prior 6 days. Visits were otherwise identical, and visit order was randomized across participants. In both cases, participants completed the following procedures during a 1.5-hour laboratory visit: two computer tasks assessing cognitive skills relevant to pedestrian safety [psychomotor vigilance test (PVT) and Conners' continuous performance task (CPT)], two brief self-report surveys on sleepiness (Epworth Sleepiness Scale and Stanford Subjective Sleepiness Scale), and 20 crossings within a virtual reality pedestrian environment. Demographic surveys were also collected.

\section{Measures}

Participants completed two cognitive tasks during each experimental visit. First, the PVT [11] was administered to evaluate reaction time, vigilance and alertness. During the test, participants responded to a red light flashing across a black computer screen. Three outcome scores were collected from this computer assessment: 1) hit reaction time, which measures the amount of time in seconds between the response stimulus appearing on the screen and the participant responding via the computer keyboard; 2) lapse count, which provides a numerical score indicating the number of times the stimulus appeared without a participant response; and 3) false starts, which refers to a numerical count of how many times the participant responded to the indicated stimuli without the stimuli appearing on the screen.

Children also completed the Conners' CPT (version 3.0, MultiHealth Systems, Inc., Toronto, Canada) to evaluate attention and response inhibition. During this 14-minute computer test, participants were instructed to hit the spacebar when any letter aside from " $X$ " appeared. For the purpose of this study, four scores were collected from this measure: 1) percentage of omissions, which refers to the number of times during the test that the participant failed to press the spacebar when they should have; 2) percentage of commissions, which refers to the number of times during the test that the participant correctly pressed the spacebar; 3) perseverations, which is a measure of random or anticipatory spacebar responses; and 4) hit reaction time score, which represents an average measure of time between the stimulus appearing on the screen and the participant pressing the spacebar.

We also administered three brief written surveys. First, a brief 
demographic survey was completed by parents during the first visit. Second, the Epworth Sleepiness Scale (completed independently by parent and child) [12] and the Stanford Sleepiness Scale (completed by child only) [13] were collected at each visit. The Epworth Sleepiness Scale consists of eight questions on a scale of zero to three and provides a general level of daytime sleepiness through self-ratings of how likely respondents feel they (or their children) are to fall asleep in a range of situations. Test-retest reliability and item analysis indicate high levels of consistency with clinical and non-clinical populations [14]. The Stanford Sleepiness Scale is a single item consisting of an eight-point scale to quantify a participants' immediate current level of sleepiness [13]. This scale has strong reliability with other sleep scales and established reliability and validity $[15,16]$.

Finally, children completed multiple crossings in a validated virtual reality pedestrian environment [17]. This environment simulates a real world street crossing by allowing children to watch and hear virtual street traffic on three consecutive monitors arranged around the child to create a semi-immersive environment. While watching traffic, participants stepped down off a wooden curb onto a pressure plate connected to the computer. This step indicated a crossing, during which a gender- and racematched avatar walked across the virtual crosswalk at the average walking pace of the participant (as measured earlier). Traffic speed for each crossing was set at a moderate speed of 32.5 miles per hour with an average traffic density of 12 vehicles per minute.

Three pedestrian safety outcome measures were derived. First, we considered collisions ("hits"), which refers to the number of times the participant was struck by a virtual vehicle across the 20 pedestrian crossings. Second, we assessed time to contact, or the average gap in seconds between the virtual pedestrian avatar and the nearest oncoming vehicle at any point in the crosswalk. Third, we examined missed opportunities, which were calculated as a count of times across the 20 trials there was a gap in traffic large enough for the participant to safely cross the street without the participant initiating a crossing. This count was quantified as a gap in traffic 1.5 times the required time for the participant to cross the street, based on their average walking speed.

\section{Data Analysis Plan}

Demographic statistics were examined first. Inspection of normality prior to inferential analyses revealed five outliers \pm 2 SD from the mean, one datapoint each from PVT mean reaction time medicated, PVT mean reaction time unmedicated, CPT perseverations medicated, CPT perseverations unmedicated, and pedestrian safety missed opportunities unmedicated. These variables were removed from the dataset prior to conducting inferential analyses. Hits in the virtual pedestrian crossing showed a non-normal distribution even after outliers were removed, so it was transformed into a binary categorical variable of any hits versus no hits across the 20 virtual crossings at each visit.

To assess the primary hypotheses, paired samples t-tests were conducted to examine differences between the within-subjects groups on the sleep, cognitive, and pedestrian outcome measures. Hits in the virtual pedestrian environment was compared across conditions using chi-square. All analyses were conducted using IBM SPSS Statistics for Windows (IBM Corp., Armonk, NY, USA).

\section{RESULTS}

We first considered parent and child reports of sleepiness in both conditions. Table 1 lists descriptive data as well as results from paired samples t-tests. As shown, children reported no significant change on the Epworth Sleepiness Scale in their self-reported general level of sleepiness while medicated $(M=11.00$, $\mathrm{SD}=3.07)$ versus not medicated $(\mathrm{M}=12.00, \mathrm{SD}=4.05), \mathrm{t}(11)=$ 0.84 . However, parents reported that their children's sleepiness improved significantly while the children were medicated $(\mathrm{M}=$ $12.75, \mathrm{SD}=4.59)$ compared to not medicated $(\mathrm{M}=16.17, \mathrm{SD}=$ 3.43), $\mathrm{t}(11)=4.87, \mathrm{p}<0.01$. Results from the self-reported Stanford Sleepiness Scale measuring the immediate level of sleepiness decreased slightly while children were medicated $(\mathrm{M}=2.50$, $\mathrm{SD}=1.62)$ versus non medicated $(\mathrm{M}=2.79, \mathrm{SD}=1.30)$, indicating a lower degree of sleepiness at the time of assessment while medicated, but this result was not statistically significant, $\mathrm{t}(11)=0.47$

Table 1 also lists descriptive data and paired sample t-test results from the cognitive measures. As shown, results from the PVT indicate the number of false starts decreased while children were medicated $(\mathrm{M}=4.00, \mathrm{SD}=2.53)$ versus not medicated $(\mathrm{M}=11.45, \mathrm{SD}=12.70), \mathrm{t}(10)=2.07, \mathrm{p}<0.10$, suggesting increased attention, vigilance, and alertness while treated with modafinil. The two other PVT outcomes, mean reaction time and lapses in attention, showed trends toward better performance while medicated, but these differences were not statistically significant.

Scores on the Conners' CPT also improved while children were medicated. In particular, the number of omissions-or failures to respond to stimuli-decreased significantly when participants were medicated $(\mathrm{M}=6.58, \mathrm{SD}=5.24)$ compared to not medicated $(\mathrm{M}=10.96, \mathrm{SD}=8.63), \mathrm{t}(11)=2.58, \mathrm{p}<0.05$. Scores on the other three measures-the percentage of commissions, number of perseverations, and hit reaction time-all improved in the hypothesized direction while participants were medicated, but at non-significant levels (Table 1).

Last, we examined the influence of treatment with modafinil on children's pedestrian safety. Results of a chi-square analysis indicate the number of collisions between participants and virtual cars improved significantly while children were treated with modafinil (25\% of participants experienced a virtual collision) compared to when they were not treated (50\% experienced a collision; $\left.\chi^{2}=4.00, \mathrm{p}<0.05\right)$. There was also significantly greater safety in time to contact, or the amount of time between the 
Table 1. Results of repeated measures t-test analyses for sleepiness, cognitive, and pedestrian measures $(n=12)$

\begin{tabular}{|c|c|c|c|c|}
\hline & Unmedicated mean (SD) & Medicated mean (SD) & $t^{*}$ & Cohen's d \\
\hline \multicolumn{5}{|l|}{ Sleepiness } \\
\hline Self-report Epworth Sleepiness Scale & $12.00(4.05)$ & $11.00(3.07)$ & 0.84 & 0.24 \\
\hline Parent-report Epworth Sleepiness Scale & $16.17(3.43)$ & $12.75(4.59)$ & $4.87 \S$ & 1.41 \\
\hline Stanford Sleepiness Scale & $2.79(1.30)$ & $2.50(1.62)$ & 0.47 & 0.14 \\
\hline \multicolumn{5}{|l|}{$\mathrm{PVT}^{\dagger}$} \\
\hline Mean reaction time & $488.38(251.33)$ & $417.32(144.93)$ & 0.75 & 0.25 \\
\hline Lapse count & $24.00(19.62)$ & $22.55(21.68)$ & 0.23 & 0.07 \\
\hline False start count & $11.45(12.70)$ & $4.00(2.53)$ & $2.07^{\| \prime}$ & 0.62 \\
\hline \multicolumn{5}{|l|}{ Conners' CPT } \\
\hline Percent of omissions & $10.96(8.63)$ & $6.58(5.24)$ & $2.58^{* *}$ & 0.74 \\
\hline Percent of commissions & $54.78(19.79)$ & $63.76(23.63)$ & -1.25 & -0.36 \\
\hline Perseverations & $1.64(2.15)$ & $0.93(1.29)$ & 1.59 & 0.50 \\
\hline Hit reaction time & 458.09 (133.93) & $422.34(77.88)$ & 1.53 & 0.44 \\
\hline \multicolumn{5}{|l|}{ Pedestrian behaviors } \\
\hline Hits & $0.77(0.93)$ & $0.50(1.00)$ & $4.00^{\ddagger * *}$ & $\mathrm{p}=0.046$ \\
\hline Time to contact & $3.94(0.72)$ & $4.31(0.74)$ & $-2.74^{* *}$ & -0.79 \\
\hline Missed opportunities & $2.00(1.79)$ & $2.55(2.46)$ & -0.73 & -0.22 \\
\hline
\end{tabular}

*One outlier \pm 2 SD from the mean was removed from analysis for each of the following variables: PVT mean reaction time medicated, PVT mean reaction time unmedicated, CPT perseverations medicated, CPT perseverations unmedicated, and pedestrian safety missed opportunities unmedicated. ${ }^{\dagger}$ Data for one participant were unavailable on this test due to technical issues, so $\mathrm{n}=11$ for this measure. ${ }^{\ddagger} \mathrm{Chi}$-square test performed for this variable, which was transformed to hits versus no hits across the 20 virtual pedestrian crossings for analysis. ${ }^{\S} \mathrm{p}<0.01$. " $\mathrm{p}<$ $0.10 .{ }^{* *} \mathrm{p}<0.05$.

SD: standard deviation, PVT: psychomotor vigilance test, CPT: continuous performance task.

pedestrian and the next approaching vehicle while medicated $(\mathrm{M}=4.31, \mathrm{SD}=0.74)$ versus not medicated $(\mathrm{M}=3.94, \mathrm{SD}=0.72)$, $\mathrm{t}(11)=-2.74 ; \mathrm{p}<0.05$. The number of missed opportunities to cross safely showed minimal change in response to the treatment with modafinil.

\section{DISCUSSION}

Use of the stimulant medication modafinil improved several aspects of cognitive functioning and pedestrian safety among children with EDS. Other aspects of functioning improved somewhat based on quantitative trends and effect sizes, although at non-significant levels among the small sample size of patients recruited for the study.

Results from the cognitive testing suggest children who were medicated experienced increased performance on measures of awareness, vigilance, and response latencies. On the PVT, the strongest result emerged on the false start count, a measure of children responding to stimuli that they should not have. The score decreased from a mean of over 11 incorrect responses to just 4 when children were medicated. On the CPT, similarly, children experienced the greatest benefit on the measure of omissions-times when they should have responded to a stimuli but did not respond. That count decreased from an average of $10.96 \%$ of the time to an average of $6.58 \%$ of the time when medicated. There also were substantial but non-significant changes (effect sizes > 0.35) on the CPT for the percent of commissions (correct responses to stimuli) and to the hit reaction time, a measure of how quickly children responded to stimuli.

The findings from the cognitive tests largely replicated in the results from the virtual reality pedestrian crossings. Half the children experienced a virtual collision in the simulated environment while unmedicated, but that number reduced to just one-quarter of children when they were medicated. This result indicates a 50\% drop in highly-dangerous pedestrian crashes for children medicated with modafinil. We also detected a change in time to contact, or the shortest time between the child pedestrian in the crosswalk and an oncoming vehicle from either direction. Time to contact reflects efficient processing and entry into a safe pedestrian gap; when it is short, the child may be choosing a risky time to cross. Thus, just as responses to the PVT and CPT while unmedicated and sleepy reflect deficiencies in cognitive skill and executive function, behavior in the virtual environment while unmedicated indicate riskier decisions. While medicated, children responded more quickly to the cognitive tasks and made safer pedestrian decisions, indications that they may have been experiencing increased attention and superior cognitive process- 
ing while medicated.

Our results concerning pedestrian behavior should be interpreted with two caveats in mind. First, the sample ranged in age from 8 years to 16 years. We might expect the youngest participants to still be developing safe pedestrian skills; the American Academy of Pediatrics suggests children under age 10 should not engage independently in street-crossing behavior [18]. The few young children in the sample, therefore, may have made frequent errors in their pedestrian crossings even when medicated, as healthy and typically-developing 8- and 9-year-old children would be expected to make poor decisions in pedestrian settings [3]. The oldest children in the sample, contrarily, may engage safely in traffic rather automatically, and therefore may suffer relatively little from their EDS symptoms. Second, children in our sample may have lived with symptoms of excessive sleepiness for many years and may therefore have developed coping skills to handle cognitively complex tasks such as street-crossing in bursts of attentive energy [19]. There is additional evidence for maturational differences when examining an increase of homeostatic sleep pressure following a full night of sleep deprivation. Older adolescents may have a higher biologic threshold to tolerate sleep deprivation symptoms than children and younger adolescents [20,21].

Study limitations include a small sample size with a wide age range. This is not unusual for research with clinically-diagnosed children with EDS given the low base rate of relevant disorders, but results should be interpreted cautiously and effect sizes considered along with statistical significance due to the available statistical power to detect differences. The wide range of ages among recruited participants also limits the study given the rapid cognitive development that occurs during the pre-teen and teen years. We examined functioning after at least six days of treatment with modafinil, which should be sufficient to induce full neurocognitive effects of the stimulant medication [22], but it is possible that longer-term results would differ if we assessed functioning repeatedly or after several weeks of administration. Finally, we conducted a substantial number of statistical tests without controlling for Type I error risk. Future research might consider ways to assess outcomes through alternative measures and recruit a larger sample size that provides sufficient statistical power to better control for Type I error risk.

Despite these limitations, we observed significant and trending patterns indicating the positive influence of the stimulant medication modafinil among children experiencing EDS. We attribute the positive changes to increased alertness, vigilance, attention, and response time and conclude treatment of EDS with stimulants like modafinil may yield improved functioning, including in life-threatening daily situations such as crossing a street.

\section{Acknowledgments}

Thanks to Anna Johnston and the UAB Youth Safety Lab for assistance in data collection and entry. Additional thanks to the Pediatric Sleep Disorders Center at Children's of Alabama for their cooperation with this research program. This project was sponsored primarily by the Kaul Pediatric Research Institute and the Alabama Children's Hospital Foundation. The content is solely the responsibility of the authors and does not necessarily represent the official views of the Kaul Pediatric Research Institute or the Alabama Children's Hospital Foundation. The project was also supported in part by the Eunice Kennedy Shriver National Institute of Child Health \& Human Development of the National Institutes of Health under Award Number R01HD088415. The content is solely the responsibility of the authors and does not necessarily represent the official views of the National Institutes of Health.

\section{Conflicts of Interest}

The authors have no financial conflicts of interest.

\section{Authors' Contribution}

Conceptualization: Avis KT, Schwebel DC. Data curation: Rouse JB. Formal analysis: Rouse JB. Funding acquisition: Avis KT, Schwebel DC. Investigation: Rouse JB, research assistants. Methodology: all authors. Project administration: Avis KT, Schwebel DC. Resources: Avis KT, Schwebel DC. Supervision: Avis KT, Schwebel DC. Writing—original draft: Rouse JB, Schwebel DC. Writing—review \& editing: all authors.

\section{REFERENCES}

1. Centers for Disease Control and Prevention, National Center for Injury Prevention and Control. WISQARS ${ }^{\mathrm{TM}}$-- web-based injury statistics query and reporting system [cited 2020 Dec 1]. Available from: https:// www.cdc.gov/injury/wisqars/index.html.

2. Barton BK, Morrongiello BA. Examining the impact of traffic environment and executive functioning on children's pedestrian behaviors. Dev Psychol 2011;47:182-91.

3. Barton BK, Schwebel DC. The roles of age, gender, inhibitory control, and parental supervision in children's pedestrian safety. J Pediatr Psychol 2007;32:517-26

4. Avis KT, Gamble KL, Schwebel DC. Does excessive daytime sleepiness affect children's pedestrian safety? Sleep 2014;37:283-7.

5. Moore M, Meltzer LJ. The sleepy adolescent: causes and consequences of sleepiness in teens. Paediatr Respir Rev 2008;9:114-21.

6. Vernet C, Leu-Semenescu S, Buzare MA, Arnulf I. Subjective symptoms in idiopathic hypersomnia: beyond excessive sleepiness. J Sleep Res 2010;19:525-34

7. Bayard S, Croisier Langenier M, Cochen De Cock V, Scholz S, Dauvilliers Y. Executive control of attention in narcolepsy. PLoS One 2012;7: e33525.

8. Calhoun SL, Fernandez-Mendoza J, Vgontzas AN, Mayes SD, Tsaoussoglou M, Rodriguez-Muñoz A, et al. Learning, attention/hyperactivity, and conduct problems as sequelae of excessive daytime sleepiness in a general population study of young children. Sleep 2012;35:627-32.

9. Ivanenko A, Tauman R, Gozal D. Modafinil in the treatment of excessive daytime sleepiness in children. Sleep Med 2003;4:579-82.

10. Sasai T, Inoue Y, Komada Y, Sugiura T, Matsushima E. Comparison of clinical characteristics among narcolepsy with and without cataplexy and idiopathic hypersomnia without long sleep time, focusing on HLA-DRB1(*)1501/DQB1(*)0602 finding. Sleep Med 2009;10:961-6.

11. Thorne DR, Johnson DE, Redmond DP, Sing HC, Belenky G, Shapiro JM. The Walter Reed palm-held psychomotor vigilance test. Behav Res Methods 2005;37:111-8.

12. Johns MW. A new method for measuring daytime sleepiness: the Epworth Sleepiness Scale. Sleep 1991;14:540-5.

13. Hoddes E, Dement WC, Zarcone V. The history and use of the Stanford Sleepiness Scale. Psychophysiology 1972;9:150.

14. Johns MW. Reliability and factor analysis of the Epworth Sleepiness Scale. Sleep 1992;15:376-81.

15. Hoddes E, Zarcone V, Smythe H, Phillips R, Dement WC. Quantification of sleepiness: a new approach. Psychophysiology 1973;10:431-6. 
16. Herscovitch J, Broughton R. Sensitivity of the Stanford Sleepiness Scale to the effects of cumulative partial sleep deprivation and recovery oversleeping. Sleep 1981;4:83-92.

17. Schwebel DC, Gaines J, Severson J. Validation of virtual reality as a tool to understand and prevent child pedestrian injury. Accid Anal Prev 2008; 40:1394-400.

18. Committee on Injury, Violence, and Poison Prevention; American Academy of Pediatrics. Policy statement--Pedestrian safety. Pediatrics 2009;124:802-12.

19. Dewald JF, Meijer AM, Oort FJ, Kerkhof GA, Bögels SM. The influence of sleep quality, sleep duration and sleepiness on school perfor- mance in children and adolescents: a meta-analytic review. Sleep Med Rev 2010;14:179-89.

20. Jenni OG, Achermann P, Carskadon MA. Homeostatic sleep regulation in adolescents. Sleep 2005;28:1446-54.

21. Taylor DJ, Jenni OG, Acebo C, Carskadon MA. Sleep tendency during extended wakefulness: insights into adolescent sleep regulation and behavior. J Sleep Res 2005;14:239-44.

22. Minzenberg MJ, Carter CS. Modafinil: a review of neurochemical actions and effects on cognition. Neuropsychopharmacology 2008;33:1477502. 